\title{
The frequency, risk factors, and complications of gastrointestinal dysfunction during enteral nutrition in critically ill patients
}

This article was published in the following Dove Press journal:

Therapeutics and Clinical Risk Management

\section{Ayse Gulsah Atasever ${ }^{\prime}$ \\ Perihan Ergin Ozcan ${ }^{2}$ \\ Kamber Kasali ${ }^{3}$ \\ Taner Abdullah ${ }^{4}$ \\ Gunseli Orhun² \\ Evren Senturk ${ }^{5}$}

'Anesthesiology and Intensive Care, Sinop Ayancik State Hospital, Sinop, Turkey; ${ }^{2}$ Anesthesiology and Intensive Care, Istanbul University Hospital, Istanbul, Turkey; ${ }^{3}$ Biostatistics, Ataturk University, Erzurum, Turkey; ${ }^{4}$ Anesthesiology Department, Istanbul University Hospital, Istanbul, Turkey;

${ }^{5}$ Anesthesiology and Intensive Care, Koc University Hospital, Istanbul, Turkey
Correspondence: Evren Senturk Intensive Care Unit, Koc University Hospital, Topkapı Street, Istanbul 34010 , Turkey

Tel +905323926633

Email evrensenturk2@yahoo.com
Background: Gastrointestinal (GI) motility disorders in intensive care patients remain relatively unexplored. Nowadays, the frequency, risk factors and complications of GI dysfunction during enteral nutrition (EN) become more questionable.

Aim: To evaluate the frequency, risk factors and complications of GI dysfunction during EN in the first 2 weeks of the intensive care unit (ICU) stay and to identify precautions to prevent the development of GI dysfunction and avoid complications.

Methods: In this prospective observational study, we deliberately targeted at-risk patients. A total of 137 patients who received nasogastric tube feeding in an ICU of a tertiary hospital were enrolled.

Results: The incidence of GI dysfunction that was found to be $63 \%$ which was associated mainly between MDR bacteria positivity and negative fluid balance. Diarrhea was observed in 36 patients (26\%) and on 147 patient-days (incidence rate, 5.5 per 100 patient-days). The median day of diarrhea onset was 6 days after the initiation of EN. Forty patients (29\%) presented with constipation ( $85 \%$ during the first week). Fifty patients (36\%) exhibited upper digestive intolerance on 212 patient-days (incidence rate, 7.9 per 100 patient-days), after a median EN duration of 6 days (range, 2-14 days). Logistic regression analysis revealed MDR bacteria growth in the culture (OR, $1.75 ; 95 \% \mathrm{CI}, 1.15-2.67 ; \mathrm{P}=0.008)$ and negative fluid balance $(\mathrm{OR}, 0.57 ; 95 \% \mathrm{CI}$, $0.34-0.94 ; P=0.03$ ) as the risk factors for GI dysfunction. We also showed that GI dysfunction was associated with high SOFA score, hypo-albuminemia, catecholamine use, and prolonged length of stay (LOS). GI dysfunction, on the other hand, can cause some complications including inadequate nutrition, and newly developed decubitus ulcers.

Conclusion: GI dysfunction should be considered a clinical predictor of inadequate nutrition and prolonged LOS. In addition, the most dramatic risk for GI dysfunction was observed in patients with MDR bacteria growth in the culture and patients in negative fluid balance. Intensivists provide appropriate nutrition for patients, as well as prompt intervention and the development of treatment strategies in the event of GI dysfunction.

Keywords: intensive care units, enteral nutrition, water-electrolyte balance, drug resistance, multiple, bacterial, gastrointestinal motility, length of stay

\section{Introduction}

Critical illness is typically associated with a catabolic stress state in which patients demonstrate a systemic inflammatory response coupled with complications of increased infectious morbidity, multiple organ dysfunction, prolonged hospitalization, and disproportionate mortality. ${ }^{1-5}$ Suspension of feeding and the resultant inability to reach nutritional goals is one complication of gastrointestinal (GI) dysfunction, but there 
are others (mucosal barrier disruption, altered motility, atrophy of the mucosa, and reduced mass of gut-associated lymphoid tissue) that may explain the greater length of stay (LOS) and death rate with GI dysfunction. ${ }^{6}$ In Europe and the United States, nutritional administration guidelines recommend primarily enteral nutrition (EN) for hemodynamically stable intensive care unit (ICU) patients. Providing EN in these patients has been shown to be superior to parenteral nutrition. ${ }^{6,7}$ GI complications such as constipation, delayed gastric emptying, diarrhea, and vomiting may occur in up to $50 \%$ of mechanically ventilated patients and adversely affect ICU mortality and LOS. ${ }^{8-12}$ Nevertheless, there is no consensus for obtaining a precise assessment of GI function. ${ }^{13}$

Diagnosis of GI dysfunction in ICU patients is complex and relies on clinical symptoms. Lack of validated markers of GI system dysfunction is often misdiagnosed and poorly managed in the ICU. ${ }^{14}$ The role of nutrition in critical illness is important, but there is an increasing evidence and broadening consensus that aggressive early feeding as well as prolonged underfeeding both should be avoided. ${ }^{15-17}$ Avoidance of complications like malnutrition, aspiration of gastric contents, wound infections, and decubitus through GI dysfunction is an important part of management of patients with GI failure.

Our hypothesis depends on the idea that GI dysfunction could be detected more than we assumed in enterally fed ICU patients, and dysfunction of the GI tract results in some problems, of which if we aim to find the most relevant risk factors for GI dysfunction, there would be a possibility to reduce them. Hence, intensivists provide appropriate nutrition for patients, as well as prompt intervention and the development of treatment strategies in the event of GI dysfunction. This prospective observational study aims to evaluate the risk of GI dysfunction and to determine the frequency, risk factors, and complications during EN in the first 2 weeks of the ICU stay.

\section{Materials and methods Patient data}

In this prospective observational study, 137 out of 226 patients consecutively admitted into a mixed medical-surgical ICU from 1 January 2015 to 31 June 2015 were enrolled according to the following criteria: ICU stay $>48$ hours, no admission diagnosis of GI bleeding, and absence of enterostomy or colostomy (Figure 1). Patients with symptoms occurring during the first 48 hours following ICU admission or those in whom laxative drugs were used, those in the prone position, or those with Clostridium difficile infection were excluded.

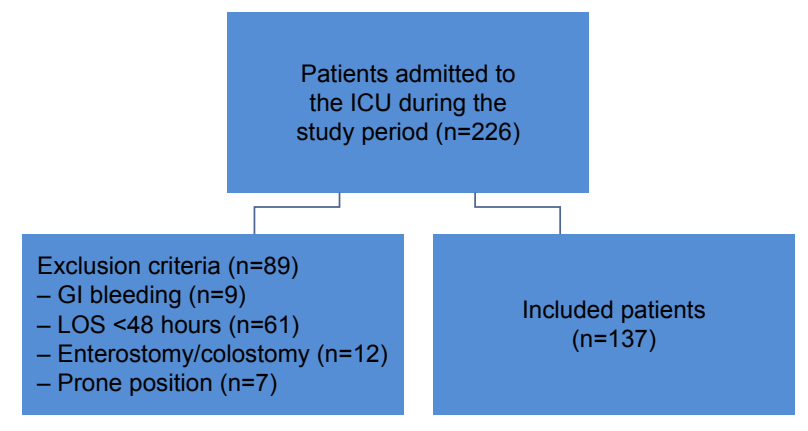

Figure I Flowchart of the study cohort.

Abbreviations: ICU, intensive care unit; GI, gastrointestinal; LOS, length of stay.

Ethical approval for the audit was obtained from the Istanbul University Clinical Research Ethics Committee (file number: 2014/1870), and written informed consent was obtained from the study subjects.

We have defined GI dysfunction as one of the following three symptoms: constipation, upper digestive intolerance (UDI), or diarrhea. Diarrhea was defined as the elimination of at least three liquid stools per day. Constipation was defined as a frequency of feces evacuation of $<3$ times per week, a feeling of incomplete rectal evacuation, hard stool or difficult passage of stool, or need for laxatives. UDI was recognized when the gastric aspirate volume (GAV) was $150-500 \mathrm{~mL}$ on two consecutive measurements, when the GAV was $>500 \mathrm{~mL}$, or when vomiting occurred. We recorded the patient age, sex, body mass index (BMI), feeding goal, sequential organ failure assessment (SOFA) score, acute physiology and chronic health evaluation II (APACHE II) score, LOS in the ICU, diagnosis, outcome, and incidence and duration of GI dysfunction episodes. During the first 2 weeks in the ICU, we recorded the number of stools per day; type of EN product administered; amount of energy delivered by EN; and development of vomiting and basic treatment agents used, such as antibiotics, antivirals, antifungals, laxatives, prokinetics, probiotics, sedatives, vasopressors, and immunosuppressants. Possible predisposing risk factors for GI dysfunction, including nosocomial pneumonia, newly developed decubitus ulcer, multidrug-resistant (MDR) bacteria-positive culture, bacteremia, sum of the fluid balance, potassium levels, $\mathrm{pH}$ levels, albumin levels, mechanical ventilation, and continuous renal replacement therapy were recorded daily until ICU discharge. The patient's status (alive or dead) on ICU discharge and length of ICU stay were recorded.

\section{Feeding protocol}

The energy target was defined as that recommended in the American Society for Parenteral and Enteral Nutrition 
guidelines, that is, $25 \mathrm{kcal} / \mathrm{kg} / \mathrm{d} .^{3}$ The feeding policy in our unit consists of starting EN via a nasogastric tube as soon as possible in all patients. The attending physician inserted a 16-Fr silicone nasogastric tube (B1cakc1lar, Istanbul, Turkey) in patients expected to stay in the ICU for longer than 48 hours. The correct position of the nasogastric tube was confirmed by injecting $40 \mathrm{~mL}$ air with a syringe down the tube and auscultating the epigastric area or by radiography if necessary. EN was initiated by the attending physician on day 1 after ICU admission in all patients who had a functional GI tract and were unable to eat orally. Anamnestic body weight was used to calculate daily energy requirements, and patient body weight was measured every 4 days. EN was administered continuously at a constant rate by using a peristaltic pump. The administration rate was increased gradually from $20 \mathrm{~mL} / \mathrm{h}$ to the appropriate rate for the individual patient. Patients were cared for in a semirecumbent position (angle of at least $30^{\circ}$ ) if the patient's hemodynamic status was stable. GAV was measured by aspirating with a $50 \mathrm{~mL}$ syringe before starting EN and every 4-6 hours from days 1 to 14 or until the end of EN. Aspirate was returned to the patient unless it exceeded $500 \mathrm{~mL}$.

\section{Statistical analysis}

The sample size was calculated to determine the frequency of diarrhea, constipation, UDI, and variables (10 variables) as the primary output criterion. Baseline characteristics are presented as mean \pm standard deviation or median (minimum-maximum) for quantitative variables, and as number (percentage) for qualitative variables. Quantitative variables were compared using an unpaired Student's $t$-test. Categorical data were compared using a $\chi^{2}$ test with Yates' correction or Fisher's exact test, depending on sample sizes. The variables "antibiotics," "antifungal drugs," "immunosuppressants," "sedatives," and "catecholamines" were analyzed according to their presence or absence daily. The variable "probiotics" was not analyzed because only one patient received probiotics. Only the first 14 days after ICU admission were considered for analysis because our study aimed to assess the GI dysfunction risk factors during the early phase of the ICU stay. Diarrhea and UDI were each analyzed according to the incidence (calculated as the number of patients with at least 1 day of either dysfunction over the total number of patients included, expressed as a percentage) and the number of affected days (calculated as the number of days with either diarrhea or UDI over the total number of analyzed days during the study period, expressed as the number of days per 100 patient-days). In addition, catecholamine use and albumin, potassium, and $\mathrm{pH}$ values (expressed per 100 patient-days) were compared with a $\chi^{2}$ test. Continuous data were compared using the Student's $t$-test. Multivariate analysis was conducted using logistic stepwise regression. Continuous data were transformed into categorical data using a clinically relevant value whenever possible or the median as the cut-off value. Data included in the logistic equation with a $P$-value $<0.05$ were considered to be independent risk factors. Data analysis was performed using SPSS 21 software (IBM Corp., Armonk, NY, USA). A $P$-value $<0.05$ was considered significant on outcome analysis.

\section{Results}

Among the 226 consecutive patients admitted to the ICU during the 6-month study period, 137 were included. There were 65 men and 72 women, with a mean age of $61.3 \pm 18.8$ years and a mean SOFA score on admission of 6.6 63 .7. Diagnostic categories are listed in Table 1. The main diagnosis for ICU admission was medical in 107 patients and surgical in 30 patients. Thirty patients had undergone recent surgery (laparotomy in 18 patients, thoracic surgery in 7 patients, and miscellaneous in 5 patients). In total, 86 patients had GI dysfunction, of whom 70 were hospitalized for medical reasons (81\%) and $16(19 \%)$ were surgical patients. The incidence of GI dysfunction in surgical and medical patients was similar (statistically not significant). Comorbidities on the first day included cardiac disease in 66 patients (48\%), diabetes mellitus in 50 patients $(36 \%)$, respiratory disease in 39 patients $(28 \%)$, renal disease in 25 patients $(18 \%)$, hematologic disease in 22 patients (16\%), and neurological disease in 34 patients $(25 \%)$.

Of the 137 patients, 86 (63\%) had GI dysfunction (Group I) for between 2 and 14 days on one or more occasion and 51 (37\%) had normal GI function (Group II). Patient characteristics according to the presence or absence of at least 1 day of GI dysfunction are listed in Table 2. Those with GI

Table I Diagnostic categories for Group I (with GI dysfunction) and Group II (without Gl dysfunction)

\begin{tabular}{lll}
\hline Diagnosis & $\begin{array}{l}\text { Group I } \\
\text { (n=86 [63\%]) }\end{array}$ & $\begin{array}{l}\text { Group II } \\
(\mathbf{n}=\mathbf{5} \text { I [37\%]) }\end{array}$ \\
\hline Medical/surgical & $70(81 \%) / 16(19 \%)$ & $37(72 \%) / 14(28 \%)$ \\
Cardiac & 46 & 20 \\
DM & 34 & 16 \\
Respiratory & 28 & 11 \\
Neurological & 18 & 16 \\
Renal failure & 16 & 9 \\
Hematologic & 14 & 8 \\
\hline
\end{tabular}

Abbreviations: GI, gastrointestinal; DM, diabetes mellitus. 
Table 2 Comparison of the two groups according to the patient characteristics at admission

\begin{tabular}{llll}
\hline & $\begin{array}{l}\text { Group I } \\
\mathbf{n = 8 6}\end{array}$ & $\begin{array}{l}\text { Group II } \\
\mathbf{n = 5} \text { I }\end{array}$ & P-value \\
\hline Age, years & $62 \pm 18$ & $60 \pm 20$ & 0.7 \\
BMI, kg/m & $27 \pm 4.7$ & $27 \pm 6.4$ & 0.7 \\
SOFA & $7.1 \pm 3.7$ & $5.9 \pm 3.7$ & 0.04 \\
APACHE II & $21 \pm 7.4$ & $21 \pm 9.9$ & 0.8 \\
\hline
\end{tabular}

Note: Group I: Patients with GI dysfunction; Group 2: Patients without GI dysfunction.

Abbreviations: $\mathrm{Gl}$, gastrointestinal; $\mathrm{BMI}$, body mass index; SOFA, sequential organ failure assessment; APACHE II, acute physiology and chronic health evaluation II.

dysfunction were characterized by a higher SOFA score at admission, high proportion of decubitus ulcers, and longer LOS in the ICU. However, age, BMI, and APACHE II score were not significantly different between the two groups.

A total of 2,693 patient-days were analyzed, and 1,132 patient-days had no GI dysfunction. During the first 2 weeks in the ICU, at least 1 day of diarrhea was observed in 30 patients (26\%). In addition, 147 days were associated with diarrhea, and the diarrhea incidence rate was 5.5 per 100 patient-days (Table 3). Constipation was seen in 40 patients (29\%), mostly in the first week (85\%). In addition, 188 days were associated with constipation, and the constipation incidence rate was 7.0 per 100 patient-days (Table 3). UDI during EN was encountered in 50 patients (36\%) at a median of 2.4 days after the onset of EN (range, 2-14 days). Furthermore, 212 days were associated with UDI, and the UDI incidence rate was 7.9 per 100 patient-days (Table 3). At least one episode of vomiting occurred in 26 patients (19\%) at a median of 1.4 days after the onset of EN (range, 2-14 days). In addition, 82 days were associated with vomiting, and the vomiting incidence rate was 3.0 per 100 patient-days (Table 3). Among 137 included patients, $C$. difficile infection was diagnosed in only two patients (overall incidence $2.2 \%$ ).

The median day of onset for both diarrhea and UDI was day 6 after admission; the median day of onset for constipation was day 3. EN was monitored for 1,196 days. The attending physician administered an antidiabetic formula to 50 diabetic patients (Resource Diabetic; Nestle, Creully,

Table 3 Epidemiology of Gl dysfunction during the first 14 days of the ICU stay (number of days [per 100 patient-days])

\begin{tabular}{ll}
\hline Number of days without GI dysfunction & $\mathrm{I}, \mathrm{I} 32(42)$ \\
Diarrhea incidence & $147(5.5)$ \\
UDI incidence & $212(7.9)$ \\
Constipation incidence & $188(7.0)$ \\
Vomiting incidence & $82(3.0)$ \\
\hline
\end{tabular}

Abbreviations: $\mathrm{Gl}$, gastrointestinal; ICU, intensive care unit; UDI, upper digestive intolerance.
Table 4 Multivariate analysis of risk factors for Gl dysfunction during EN in 137 patients in the ICU

\begin{tabular}{lllll}
\hline Variable & Wald & P-value & OR & 95\% Cl \\
\hline MDR bacteria-positive culture & 6.97 & 0.008 & 1.75 & $1.15-2.67$ \\
Negative fluid balance & 4.71 & 0.03 & 0.57 & $0.34-0.94$ \\
\hline
\end{tabular}

Abbreviations: $\mathrm{Gl}$, gastrointestinal; EN, enteral nutrition; ICU, intensive care unit; $\mathrm{OR}$, odds ratio; $\mathrm{Cl}$, confidence interval; MDR, multidrug resistant.

France or Glucerna SR; Abbott, Zwolle, Holland), a kidneyprotective formula to 25 renal failure patients (Nepro; Abbott), and a standard enteral formula to the remaining 62 patients (Jevity, Osmolite, Ensure Plus; Abbott). Most diets contained $1.2 \mathrm{kcal} / \mathrm{mL}$.

In the multivariable logistic regression model for GI dysfunction adjusted for MDR bacteria-positive culture, SOFA score, decubitus ulcers, negative fluid balance, bacteremia, and catecholamine use, a significant relationship remained between GI dysfunction and negative fluid balance (odds ratio [OR], $0.57 ; 95 \%$ confidence interval [CI], 0.34-0.94; $P=0.03$ ) and MDR bacteria growth in the culture (OR, 1.75; 95\% CI, 1.15-2.67; $P=0.008$; Table 4). In addition, high SOFA scores $(P=0.04)$, catecholamine use on at least 1 day during the ICU stay $(P<0.001)$, and albumin levels $<2.5 \mathrm{~g} / \mathrm{dL}(P=0.005)$ were revealed as risk factors for GI dysfunction.

Certain drugs used during the ICU stay were analyzed for the risk of GI dysfunction (Table 5). The highest relative risk was observed with catecholamine use. Immunosuppressants, prokinetics, laxatives, antibiotics, antifungal drugs, insulin, continuous renal replacement therapy, and mechanical ventilation were not significantly associated with the risk of GI dysfunction (Table 5). A stratified analysis was conducted to analyze the risk of GI dysfunction according to the albumin, potassium, and $\mathrm{pH}$ levels. There were no significant connections between GI dysfunction and acidosis or alkalosis and hypokalemia or hyperkalemia. However, patients with hypoalbuminemia were characterized by a higher proportion of GI dysfunction $(P<0.05)$.

Comparisons of outcome measures in patients with and without GI dysfunction during EN are listed in Table 6 .

Table 5 Treatments during EN in 137 patients in the ICU (n [\%])

\begin{tabular}{llll}
\hline Treatments & $\begin{array}{l}\text { Group I } \\
\mathbf{n = 8 6}\end{array}$ & $\begin{array}{l}\text { Group II } \\
\mathbf{n = 5} \text { I }\end{array}$ & P-value \\
\hline Catecholamines use & $50(58 \%)$ & $21(41 \%)$ & $<0.00$ I \\
Sedative drugs & $67(78 \%)$ & $37(72.5 \%)$ & 0.47 \\
Insulin & $42(48 \%)$ & $20(39 \%)$ & 0.27 \\
Antifungal drugs & $31(36 \%)$ & $13(25 \%)$ & 0.14 \\
\hline
\end{tabular}

Note: Group I: Patients with GI dysfunction; Group 2: Patients without GI dysfunction.

Abbreviations: $\mathrm{EN}$, enteral nutrition; ICU, intensive care unit; GI, gastrointestinal. 
Table 6 Comparison of outcome according to measurement of GI dysfunction during EN in 137 patients in the ICU (n [\%])

\begin{tabular}{llll}
\hline Outcomes & $\begin{array}{l}\text { Group I } \\
\mathbf{n = 8 6}\end{array}$ & $\begin{array}{l}\text { Group II } \\
\mathbf{n = 5} \text { I }\end{array}$ & P-value \\
\hline Pneumonia & $28(32.6 \%)$ & II $(21.6 \%)$ & 0.16 \\
Mortality & $43(50 \%)$ & $18(35 \%)$ & 0.094 \\
Unable to reach & $23(25 \%)$ & $2(4.4 \%)$ & 0.003 \\
$\begin{array}{l}\text { target energy intake } \\
\text { Decubitus ulcers }\end{array}$ & $45(52 \%)$ & $15(29 \%)$ & 0.009 \\
LOS & $21.4 \pm 17$ & $16 \pm 18.5$ & 0.002 \\
\hline
\end{tabular}

Note: Group I: Patients with GI dysfunction; Group 2: Patients without GI dysfunction.

Abbreviations: Gl, gastrointestinal; EN, enteral nutrition; ICU, intensive care unit; LOS, length of stay.

The LOS, number of patients with decubitus ulcers, and number of patients who could not achieve target energy intake were significantly greater in the patients with GI dysfunction than those without GI dysfunction. The median LOS per patient was 19.5 days (range, 1-103 days). In our study, enteral feeding was attempted in all patients, and only $25(18 \%)$ out of 137 patients with or without GI dysfunction failed to achieve the target energy intake. However, enteral feeding was delayed in $25 \%$ of the patients with GI dysfunction and $4 \%$ of the patients without GI dysfunction ( $P=0.003)$. GI dysfunction was not significantly associated with pneumonia or mortality.

\section{Discussion}

Our study investigates the incidence of GI dysfunction that was found to be $63 \%$, which was associated mainly between MDR bacteria positivity and negative fluid balance. In our findings, negative fluid balance and MDR bacteria positivity were independent risk factors for GI dysfunction. We also showed that GI dysfunction was associated with high SOFA score, hypoalbuminemia, catecholamine use, and prolonged LOS. GI dysfunction, on the other hand, can cause some complications including inadequate nutrition, vomiting, and newly developed decubitus ulcers, and these complications may delay patient recovery. Therefore, this study reveals the need for the establishment of a management protocol to treat these complications.

Fluid balance is important and may lead to GI dysfunction in the critically ill. We know of one meta-analysis involving 19,902 patients who reported positive fluid balance was associated with intra-abdominal hypertension and increased complications such as intestinal edema, malabsorption, bacterial translocation, ileus, acidosis, and increased intestinal permeability. ${ }^{18}$ That study described the pathophysiological effects of positive fluid balance in the GI tract, such as reduced abdominal perfusion pressure, bowel contractility, and enteral feeding success. In our study, contrary to these findings, the relationship between negative fluid balance and GI dysfunction was investigated for the first time in the literature, and negative fluid balance was found to be a risk factor for GI dysfunction. This can be explained by convective problems, disruption of intestinal perfusion, and tissue hypoxia, resulting in intestinal motility disorders in patients with negative fluid balance. Therefore, during the period of fluid restriction, there was a significant reduction in stool weight and frequency as well as an increased tendency toward constipation, which indicates the importance of a normal state of hydration.

There is no report in the literature showing the relationship between MDR bacteria-positive patients and GI dysfunction. Therefore, our study was the first prospective observational study investigating this relationship. Results of this study showed GI dysfunction occurred more often in patients who have MDR bacteria-positive culture, and we assumed that this might have resulted from possible antibiotic usage and change in microbiome. As assumed, GI dysfunction not only might have resulted from a change in microbiome but also might be caused by a cofounding factor of vasopressor use. Since MDR bacteria-positive patients are often critically ill patients, they receive vasopressor treatment more commonly, and it should not be forgotten that vasopressor treatment is also a risk factor for GI dysfunction.

Critically ill patients are often treated with opiates, antibiotics, and catecholamines, which have already been identified as a risk factor for GI intolerance during EN. ${ }^{19,20}$ In a study conducted by Mentec et al, ${ }^{20}$ the incidence of upper GI dysfunction was increased in patients receiving sedative and vasopressor medication. Furthermore, reduction in the dosage of opiates and catecholamines may help reduce exogenous causes of delayed gastric emptying. ${ }^{21-23}$ Similarly, catecholamines were independently associated with GI dysfunction in our study, but, in contrast to the findings of previous studies, we found similar use of opiates in patients with and without GI dysfunction. The rationale is to improve splanchnic perfusion to prevent or overcome dysfunction and to preserve the intestinal microbiome. ${ }^{24}$

In critical illness, the interpretation of low citrulline as a marker of intestinal dysfunction should be treated with caution, in a similar manner in which low albumin in a critically ill patient needs to be cautiously interpreted as malnutrition. ${ }^{25}$ Citrulline is derived from the amount produced in the enterocytes of the small bowel. Levels of citrulline lower than $20 \mathrm{mmol} / \mathrm{L}$ seems to be indicative of intestinal insufficiency. 
Therefore, provision of adequate nutritional support to the critically ill is quite important to prevent malnutrition, and these biomarkers can lead us to detect related complications. However, EN in critically ill patients with GI dysfunction remains a clinical challenge.

A previous study in intensive care patients revealed that constipation caused a delay in enteral feeding in $50 \%$ of patients. ${ }^{26}$ Similarly, our study also showed that enteral feeding was significantly delayed in patients with GI dysfunction compared to those without. Therefore, management of GI dysfunction is critically important to prevent complications resulting from lack of feeding. Moreover, in the literature, the frequency of diarrhea varies between $2 \%$ and $95 \%$ in the critically ill. ${ }^{27}$ Compared to this, the incidence of diarrhea in our study might have been considered relatively low because of the differences in the definition of the condition. It is once again noteworthy that GI motility disorders lack precise definitions.

In our unit, patients were treated according to standard protocols for feeding, weaning from mechanical ventilation, sedation, and cardiovascular support. Fluid and electrolyte balance was measured daily and promptly treated and the patients were kept hemodynamically stable. We provided bowel care in the form of laxatives or prokinetic agents when signs of GI dysfunction were observed. GAVs are generally considered to indicate gastric emptying rate; volumes aspirated are also affected by the rate of feed administration, the technique of aspiration, gastric secretion, and duodenogastric reflux. ${ }^{28}$

The absence of a consensus definition of GI dysfunction is a major limiting factor of research in this area. The absence of a clear definition of GI dysfunction and the limited number of patients and the inability of measuring nutritional and inflammation biomarkers should also be considered as main limitations of this study. We consider that dehydration, limited types of EN products in our hospital pharmacy, lack of fiber in the diets, and the difficulties of providing care to patients with MDR bacteria positivity were associated with the development of GI dysfunction.

In conclusion, maintaining a normal state of hydration is important, as dehydration has been shown to be a risk factor for many health conditions. ${ }^{29}$ Moreover, this is the first report in the literature investigating GI dysfunction in patients with MDR bacteria positivity and with negative fluid balance. We concluded that GI dysfunction should be considered a clinical predictor of inadequate nutrition and prolonged LOS. In addition, the most significant risk for GI dysfunction was observed in patients with MDR bacteria growth in the culture and patients in negative fluid balance. Intensivists provide appropriate nutrition for patients, as well as prompt intervention and the development of treatment strategies in the event of GI dysfunction. In addition, further studies are needed to clarify these relationships. Finally, there is a need for more prospective studies to compare different management strategies for GI dysfunction in critically ill patients.

\section{Acknowledgment}

We thank Dr Achmet Ali (Capa clinics Istanbul, Turkey) for his help with regard to the statistical analyses.

\section{Author contributions}

All authors contributed toward data analysis, drafting and critically revising the paper and agree to be accountable for all aspects of the work.

\section{Disclosure}

The authors report no conflicts of interest in this work.

\section{References}

1. Jensen GL, Compher C, Sullivan DH, Mullin GE. Recognizing malnutrition in adults: definitions and characteristics, screening, assessment, and team approach. JPEN J Parenter Enteral Nutr. 2013;37(6):802-807.

2. Preiser JC, van Zanten AR, Berger MM, et al. Metabolic and nutritionals support of critically ill patients: consensus and controversies. Crit Care. 2015;19:35.

3. Tian F, Gao X, Wu C, Zhang L, Xia X, Wang X. Initial energy supplementation in critically ill patients receiving enteral nutrition: a systematic review and meta-analysis of randomized controlled trials. Asia Pac J Clin Nutr. 2017;26:11-19.

4. Weijs PJ, Looijaard WG, Beishuizen A, Girbes AR, Oudemans van Straaten HM. Early high protein intake is associated with low mortality and energy overfeeding with high mortality in non-septic mechanically ventilated critically ill patients. Crit Care. 2014;18:701.

5. Harvey SE, Parrott F, Harrison DA, et al. Trial of the route of early nutritional support in critically adults. $N$ Engl J Med. 2014;371: 1673-1684.

6. Taylor BE, McClave SA, Martindale RG, et al. Guidelines for the provision and assessment of nutrition support therapy in the adult critically ill patient: Society of Critical Care Medicine (SCCM) and American Society for Parenteral and Enteral Nutrition (ASPEN). Crit Care Med. 2016;44:390-438.

7. Reintam Blaser R, Starkopf J, Alhazzani W, et al. Early enteral nutrition in critically ill patients: ESICM clinical practice guidelines. Intensive Care Med. 2017;43:380-398.

8. Blaser AR, Malbrain ML, Starkopf J, et al. Gastrointestinal function in intensive care patients: terminology, definitions and management. Recommendations of the ESICM working group on abdominal problems. Intensive Care Med. 2012;38:384-394.

9. Mutlu GM, Mutlu EA, Factor P. GI complications in patients receiving mechanical ventilation. Chest. 2001;119:1222-1241.

10. Montejo JC. Enteral nutrition-related gastrointestinal complications in critically ill patients: a multicenter study: The Nutritional and Metabolic Working Group of the Spanish Society of Intensive Care Medicine and Coronary Units. Crit Care Med. 1999;27:1447-1453.

11. Montejo JC, Miñambres E, Bordejé L, et al. Gastric residual volume during enteral nutrition in ICU patients: the REGANE study. Intensive Care Med. 2010;36:1386-1393.

12. ReintamBlaser A, Jakob SM. Gastrointestinal failure in the ICU. Curr Opin Crit Care. 2016;22:128-141. 
13. Reintam A, Parm P, Kitus R, et al. Gastrointestinal failures core in critically ill patients: a prospective observational study. Crit Care. 2008;12:R90.

14. Puleo F, Arvanitakis M, Van Gossum A, et al. Gut failure in the ICU Semin Respir Crit Care Med. 2011;32:626-638.

15. Casaer MP, Ziegler TR. Nutritional support in critical illness and recovery. Lancet Diabetes Endocrinol. 2015;3:734-745.

16. Hooper MH, Marik PE. Controversies and misconceptions in intensive care unit nutrition. Clin Chest Med. 2015;36:409-418.

17. Preiser JC, Taccone FS. Nutrition in critically ill patients: where do we stand? Minerva Anestesiol. 2016;82(8):908-913.

18. Malbrain ML, Marik PE, Witters I, et al. Fluid overload, de-resuscitation, and outcomes in critically ill or injured patients: a systematic review with suggestions for clinical practice. Anaesthesiol Intensive Ther. 2014;46:361-380.

19. Nguyen NQ. Pharmacological therapy of feed intolerance in the critically ills. World J Gastrointest Pharmacol Ther. 2014;5:148-155.

20. Mentec H, Dupont H, Bocchetti M, Cani P, Ponche F, Bleichner G. Upper digestive intolerance during enteral nutrition in critically ill patients: frequency, risk factors, and complications. Crit Care Med. 2001;29:1955-1961.

21. Nguyen NQ, Chapman MJ, Fraser RJ, et al. The effects of sedation on gastric emptying and intra-gastric meal distribution in critical illness. Intensive Care Med. 2008;34:454-460.
22. Wells DL. Provision of enteral nutrition during vasopressor therapy for hemodynamic instability: an evidence-based review. Nutr Clin Pract. 2012;27:521-526.

23. Khalid I, Doshi P, DiGiovine B. Early enteral nutrition and outcomes of critically ill patients treated with vasopressors and mechanical ventilation. Am J Crit Care. 2010;19:261-268.

24. Blaser AR, Starkopf J, Malbrain ML. Abdominal signs and symptoms in intensive care patients. Anaesthesiol Intensive Ther. 2015;47(4): 379-387.

25. Fragkos KC, Forbes A. Citrulline as a marker of intestinal function and absorption in clinical settings: a systematic review and meta-analysis. United European Gastroenterol J. 2017;0(0):1-11.

26. Mostafa SM, Bhandari S, Richie G, Gratton N, Wenstone R. Constipation and its implications in the critically ill patients. Br J Anaesth. 2003; 91:815-819.

27. Thibault R, Clerc A, Delieuvin N, Heidegger CP, Pichard C. Diarrhoea in the ICU: respective contribution of feeding and antibiotics. Crit Care. 2013; 17:R153.

28. Arabi YM, Casaer MP, Chapman M, et al. The intensive care medicine research agenda in nutrition and metabolism. Intensive Care Med. 2017;43(9):1239-1256.

29. El-Sharkawy AM, Sahota O, Lobo DN. Acute and chronic effects of hydration status on health. Nutr Rev. 2015;73:97-109.
Therapeutics and Clinical Risk Management

\section{Publish your work in this journal}

Therapeutics and Clinical Risk Management is an international, peerreviewed journal of clinical therapeutics and risk management, focusing on concise rapid reporting of clinical studies in all therapeutic areas, outcomes, safety, and programs for the effective, safe, and sustained use of medicines. This journal is indexed on PubMed Central, CAS,

\section{Dovepress}

EMBase, Scopus and the Elsevier Bibliographic databases. The manuscript management system is completely online and includes a very quick and fair peer-review system, which is all easy to use. Visit http://www.dovepress.com/testimonials.php to read real quotes from published authors.

Submit your manuscript here: http://www.dovepress.com/therapeutics-and-clinical-risk-management-journal 